$16^{\text {th }}$ International Congress of Metrology, 05009 (2013)

DOI: $10.1051 /$ metrology/201305009

(c) Owned by the authors, published by EDP Sciences, 2013

\title{
Development of a new in-water-phantom graphite calorimeter for the measurement of absorbed dose to water in medium energy $x$-ray beams
}

\author{
Massimo Pinto ${ }^{1, \mathrm{a}}$, Maria Pimpinella ${ }^{1}$, Antonio Stefano Guerra ${ }^{1}$, Iordana Astefanoaei ${ }^{2}$, Stefano Loreti ${ }^{1}$, Maurizio Quini ${ }^{1}$, \\ and Maria Pia Toni ${ }^{1}$ \\ ${ }^{1}$ Istituto Nazionale di Metrologia delle Radiazioni Ionizzanti dell'ENEA (ENEA-INMRI), Via Anguillarese , 301,00123 \\ Santa Maria di Galeria (RM), Italy \\ ‘'Al I. Cuza’ Iasi University, Faculty of Physics, Carpath Center, 11 Carol I Blvd, RO-700506 Iasi, Romania
}

\begin{abstract}
This paper summarizes the progress on the construction of a graphite calorimeter for the measurement of absorbed dose to water $\mathrm{D}_{\mathrm{w}}$ in medium-energy filtered $\mathrm{x}$-ray qualities, as part of the EMRPfunded project "Metrology for radiotherapy using complex radiation fields" (MetrExtRT, http://radiotherapyemrp.eu/). The design and the construction of a new primary standard for absorbed dose to water $\left(D_{w}\right)$ is shown, together with a presentation of the preliminary estimates of conversion factors, calculated by Monte Carlo using the EGSnrc code, and heat-transport calculations resulting from COMSOL simulations. The vacuum-gap-insulated calorimeter core has a thickness of $2 \mathrm{~mm}$ and a diameter of $20 \mathrm{~mm}$. The graphite calorimeter is embedded in a PMMA waterproof sleeve and can be positioned at the desired measurement depth in a water phantom. Once completed, the new calorimeter will participate in an international comparison with other existing standards of absorbed dose to water for medium energy $\mathrm{x}$-rays.
\end{abstract}

\section{Introduction}

International reports recommend that the uncertainty in dose delivery in all modalities of radiotherapy should fall within $5 \%$ at the $95 \%$ confidence interval [dose delivered to the target volume, 1,2]. Although some radiation modalities now benefit from uncertainties that are in compliance with these recommendations, there are several other radiation modalities in which uncertainties are still larger than $5 \%$. Part of the problem lies in the lack of primary standards of absorbed dose to water $\left(D_{w}\right)$ and lack of traceability in reference dosimetry. In the absence of a primary standard for absorbed dose to water, measurements of $D_{w}$ rely on the use of instruments calibrated against other quantities (such as air kerma) and the application of dosimetry protocols. Among the radiotherapy modalities where dosimetry is not yet traceable to absorbed dose to water standards, mediumenergy x-ray beams are used for the treatment of superficial skin tumours as well as other benign diseases.

The Italian National Institute of Ionizing Radiation Metrology (ENEA-INMRI) is participating in a research project funded by the European Metrology Research Program (EMRP) called "Metrology for radiotherapy using complex radiation fields" (MetrExtRT, http://radiotherapy-emrp.eu/) that aims at improving reference dosimetry for a number of radiotherapy conditions that rely on the use of photons [3]. Among the tasks in which ENEA-INMRI is involved, we are building a new graphite calorimeter which, positioned in a water-phantom allows the realization of absorbed-doseto-water unit for medium-energy x-ray beams. This new primary standard after successful completion of an international comparison, will become the Italian Standard for absorbed dose to water $\left(D_{w}\right)$ for mediumenergy x-ray beams. This short article outlines the design of this new calorimeter and its ongoing construction. Results from $D_{w}$ measurements realized using this new calorimeter will be subject of future reports.

\section{Construction of the in-water graphite calorimeter}

The standard has been designed as a miniature three bodies (core, jacket and shield) graphite calorimeter, embedded in a PMMA evacuated envelope, allowing for measurements in a water phantom at $2 \mathrm{~g} \mathrm{~cm}^{-2}$ depth [4, see figure 1]. According to a nested design, the innermost graphite body (the disc-shaped core, $20 \mathrm{~mm}$ diameter, $2 \mathrm{~mm}$ thickness) is surrounded by a $0.5 \mathrm{~mm}$-thick vacuum gap and then by a second graphite body (the jacket) with the same heat capacity as the core. The jacket is surrounded by an additional $0.5 \mathrm{~mm}$-thick vacuum gap and then by a third graphite body (the medium) acting as a thermal buffer to improve the core thermal insulation.

\footnotetext{
${ }^{\text {a }}$ Corresponding author: massimo.pinto@enea.it
} 
Temperature variations are measured by $0.25 \mathrm{~mm}$ diameter microthermistors. The measurement of the absorbed dose to graphite, $\mathrm{D}_{\mathrm{g}}$, is made by determining the calorimeter electrical calibration coefficient (i.e. the ratio of the energy absorbed in the core to the corresponding fractional change of core thermistors resistance) and then by measuring the fractional change of the core thermistors resistance under $\mathrm{x}$-ray irradiation.

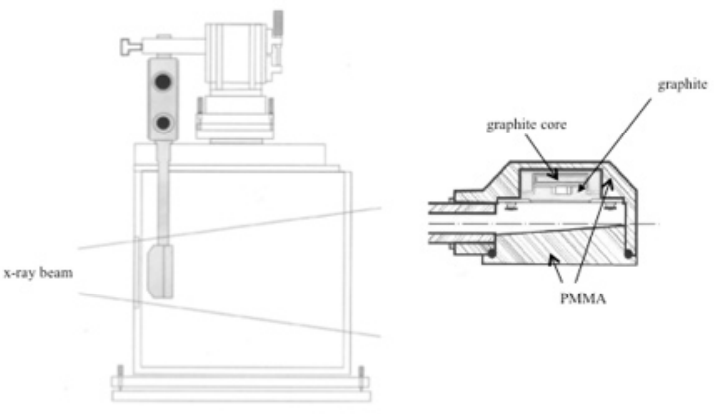

Figure 1. A Schematic view of the calorimeter positioned in the water phantom before an x-ray beam (left). On the right-hand side, a cutaway of the graphite components of the calorimeter as they are encapsulated in the PMMA evacuated housing.

The calorimeter is still under construction at the time of preparing this document. One representative photograph of the graphite elements of the calorimeter, the aluminized-Mylar-coated jacket base and the shield base, is shown in figure 2.

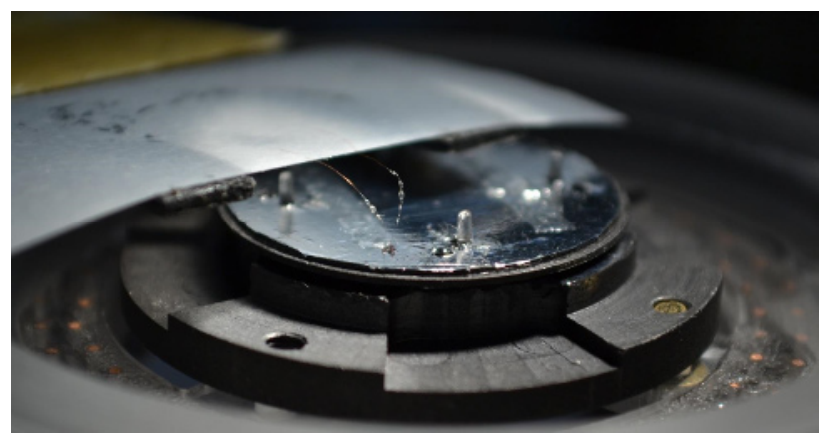

Figure 2. Assembling of the graphite components of the calorimeter. Shown in the photo is a close-up of the shield base to which the jacket base is attached, with one of the jacket base thermistor being glued inside a tiny hole drilled in graphite, underneath the aluminized Mylar layer. Thin copper wires are welded to the thermistor's Pt-Ir electrical wires, the latter insulated by beads of epoxy that also help to keep the electrical contacts in place.

\subsection{Heat-transfer calculations using the finite- elements method}

Using the finite element method (FEM) in Comsol Multiphysics software, a complete 3D model of the calorimeter was developed. The model describes the spatial distribution and the time evolution of the temperature field in the full complex geometry of the calorimeter designed at ENEA-INMRI. All the characteristics and thermal properties of the materials used in the calorimeter construction were set as input parameters into calculations. The model allowed to simulate the thermal processes (conduction and radiative heat-transfer) occurring in the calorimeter due to absorption of the ionizing radiation in the different calorimeter bodies. The convection heat losses between the calorimeter bodies and the cubic water phantom in which the calorimeter is immersed were also considered (Fig. 3).

The Monte Carlo calculations of the absorbed dose distribution in the calorimeter were used to model the heat source describing the effect of the absorption and scattering of radiation in graphite. To this aim, a simulation of the calorimeter irradiation in water was performed by EGSnrc Monte Carlo code [5]. The absorbed dose was calculated as a function of depth in water considering all the different materials of the calorimeter. The heat-source due to the energy deposition by the $\mathrm{x}$-ray radiation source was modelled by interpolation of the Monte Carlo absorbed dose distribution data. The time evolution of the spatial distribution of the temperature in graphite and PMMA was related to the absorbed dose spatial distribution via the material's specific heat capacity.

The temperature field corresponding to the calorimeter design was calculated by solving the Fourier's heat-transport equation with suitable boundary conditions. The heat-source was considered as a input file. The calorimeter pieces were considered at the temperature $298.15 \mathrm{~K}$ and the water phantom at the temperature $295.15 \mathrm{~K}$.
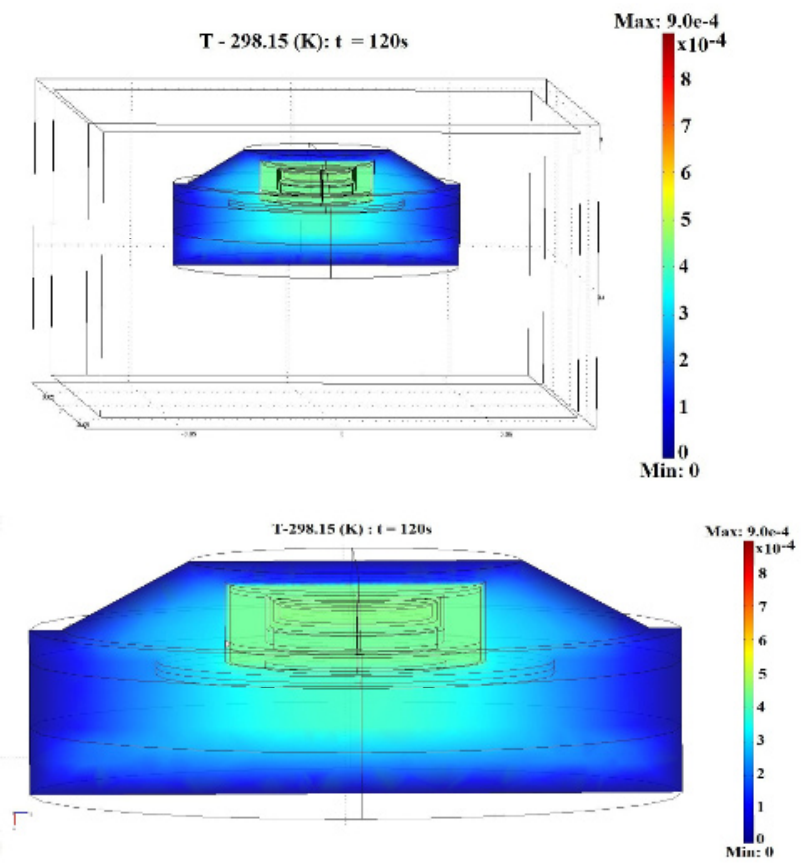

Figure 3. The spatial distribution of the temperature after a 2 min irradiation at a dose rate of $0.3 \mathrm{~Gy} / \mathrm{min}$. Top: The temperature field in calorimeter after a 2 min irradiation run at a dose rate of $0.3 \mathrm{~Gy} / \mathrm{min}$. Bottom illustration: Enlarged view of the temperature field in calorimeter after a $2 \mathrm{~min}$ irradiation run at a dose rate of $0.3 \mathrm{~Gy} / \mathrm{min}$.

To optimize the design of the calorimeter, the time evolution of the thermal gradients in the core during the irradiation process was analysed for different diameters 
of the medium using the finite element method. The thermal gradients and the time needed to reach thermal equilibrium in the core (core equilibrium time) were computed for different values of medium radius (figure 4).

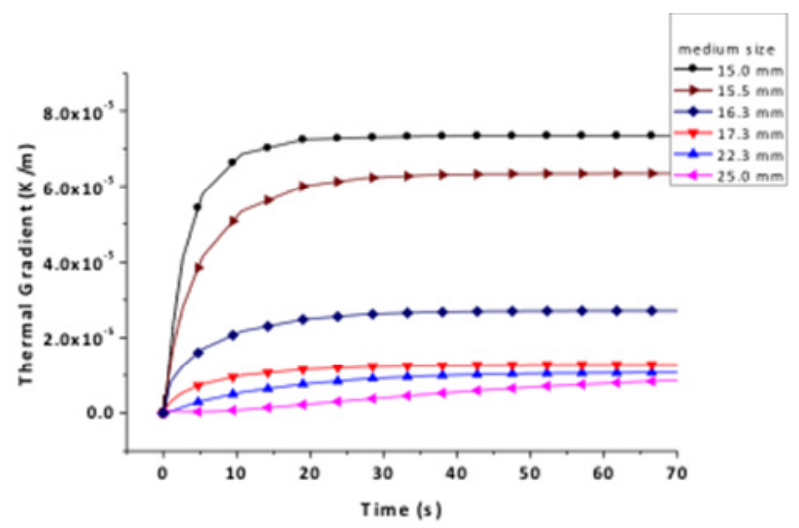

Figure 4. Time evolution of the thermal gradients in the core for different medium radii. Results from calculations using the finite element method.

As is shown in figure 4 , the medium size has an influence on thermal stability of the core. A good thermal stability of the core corresponds to small values of the core thermal equilibrium time and to small thermal gradients. If the medium size is larger than $17.3 \mathrm{~mm}$ in its radius, the heat losses by conduction and radiative heat transfer increase and the core thermal equilibrium time becomes longer. The core equilibrium time is about $25 \mathrm{~s}$ for a medium radius of $17.3 \mathrm{~mm}$. This dimension is a good compromise between the small value of thermal gradient and the core equilibrium time, and was adopted in the final calorimeter design.

The 3D temperature distribution and the 3D temperature gradients at different times due to the irradiation were calculated in the case of the calorimeter design eventually adopted. In particular, the effects of the residual air pressure in the insulating gaps were included in the model. Actually, the calorimeter is not equipped with vacuum insulating gaps, but with insulating gaps filled with air at low pressure. Therefore, the free molecular regime conduction occurs in the low-pressure air filling the gaps.

The 3D model of the temperature distribution and temperature gradients due to irradiation also allows to calculate heat-loss correction factors.

\subsection{Conversion and correction factors determined via Monte Carlo simulations}

Preliminary Monte Carlo simulations were run using the code EGSnrc [5] to evaluate the physical parameters, and the correction factors, needed to convert absorbed-dose to graphite to absorbed-dose to water. A cylindrical symmetry was used for modelling the calorimeter and its envelope in a water phantom. The XCOM photon crosssection library as distributed with the EGSnrc code [6] was adopted for the simulations. Electrons and photons were followed down to a kinetic energy of $1 \mathrm{keV}$.
Rayleigh scattering, atomic relaxations, binding effects for Compton scattering and angular sampling of photoelectrons were accounted for in the simulations.

An overall conversion factor has been introduced as:

$$
C_{w, g}=D_{w} / D_{g}
$$

where $D_{w}$ is the absorbed dose at $2 \mathrm{~cm}$ depth in homogeneous water and $D_{g}$ the absorbed dose in the calorimeter graphite core at the same depth, in water. The $\mathrm{C}_{\mathrm{w}, \mathrm{g}}(\mathrm{Q})$ conversion factor for a $\mathrm{x}$-ray beam with a given spectral distribution $\mathrm{Q}$ can be determined as:

$$
\overline{\mathrm{C}_{\mathrm{w}, \mathrm{g}}(Q)}=\sum_{\mathrm{i}}^{\mathrm{N}} \mathrm{C}_{\mathrm{w}, \mathrm{g}}\left(\mathrm{E}_{\mathrm{i}}\right) \quad \mathrm{E}_{i} \Delta \Phi_{i} / \sum_{\mathrm{i}}^{\mathrm{N}} \mathrm{E}_{i} \Delta \Phi_{i}
$$

where $\mathrm{N}$ is the number of energy bins for the photon spectral distribution, $E_{i}$ is the mean energy of the $i^{\text {th }}$ bin, $\mathrm{C}_{\mathrm{w}, \mathrm{g}}\left(\mathrm{E}_{\mathrm{i}}\right)$ is the conversion factor obtained by equation (1) using $D_{w}$ and $D_{g}$ calculated by MC for ideal mono-energetic x-rays with energy $\mathrm{E}_{\mathrm{i}}$ and $\Delta \Phi_{i}$ is the photon fluence of the beam in the $i^{\text {th }}$ energy bin. Calculated values of $C_{w, g}(E)$ in the energy range from $200 \mathrm{keV}$ to $20 \mathrm{keV}$ are shown in figure 5. To evaluate the accuracy of equation (2), $\overline{\mathrm{C}_{\mathrm{w}, \mathrm{g}}(Q)}$ values were calculated for two reference $\mathrm{x}$-ray beams available at ENEA-INMRI and compared to the corresponding conversion factors directly obtained by Monte Carlo simulations through equation (1). In this case the simulations were performed sampling the energy of incident photons according to the actual beam spectral distributions. Results indicate that $\mathrm{C}_{\mathrm{w}, \mathrm{g}}(\mathrm{E})$ values calculated with steps of $10 \mathrm{keV}$ and equation (2) allow to determine conversion factors for real $\mathrm{x}$-ray beams with an accuracy compared to that obtained performing Monte Carlo simulations for each specific spectral distribution. Due to the wide range of spectral distributions of x-ray beams the use of equation (2) has the advantage of a sudden calculation of $\mathrm{C}_{\mathrm{w}, \mathrm{g}}(\mathrm{Q})$ for any beam quality, once the $\mathrm{C}_{\mathrm{w}, \mathrm{g}}(\mathrm{E})$ factors have been calculated as a function of energy.

An expression of $\mathrm{C}_{w, g}$ as product of specific physical parameters and correction factors determined through independent simulations is under validation. Simulations mostly refer to evaluation of correction factors accounting for perturbation effects due to the presence of all the non water-equivalent materials of the calorimeter, including graphite and vacuum gaps. Moreover spectral energy fluence distributions at the measurement point in water and in the calorimeter core are calculated in order to determine the mean ratio of mass-energy absorption coefficients of water to graphite that represents the largest component of the $\mathrm{C}_{\mathrm{w}, \mathrm{g}}$ factor. 


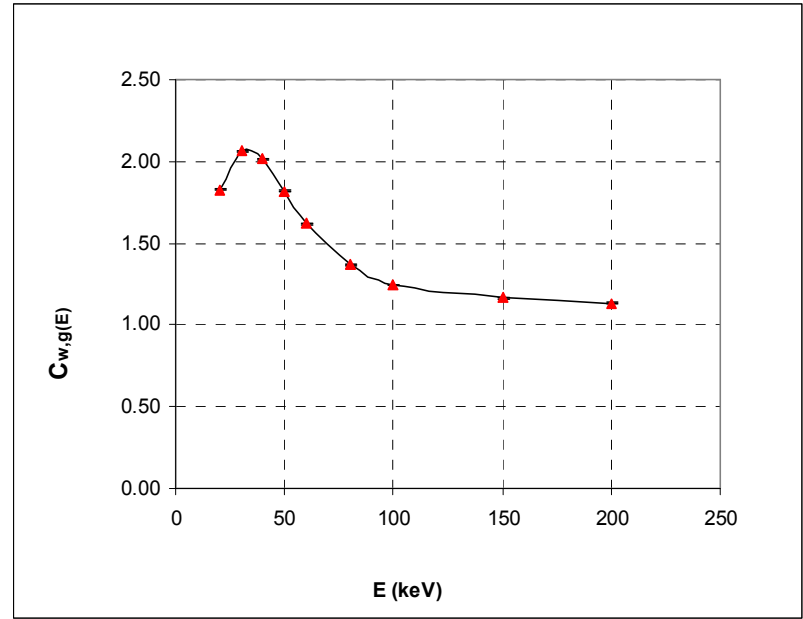

Figure 5. Conversion factors for the ENEA-INMRI calorimeter obtained by Monte Carlo simulations for monoenergetic photons in the medium-energy range. The statistical uncertainty of data is $0.3 \%$.

\section{Conclusions}

A new standard for the measurement of absorbed dose to water for medium energy $\mathrm{x}$-rays is under construction at ENEA-INMRI. The graphite calorimeter has been designed with the aid of Monte Carlo simulations and finite-element method-based calculations.

Upon completion of the calorimeter and after preliminary measurements at ENEA-INMRI, the standard will participate in an international comparison with other established standards of $\mathrm{D}_{\mathrm{w}}$ for medium energy $\mathrm{x}$-rays based on different methods (water calorimetry and ionometric method). The ENEA-INMRI standard being the first $D_{w}$ standard for medium energy x-rays based on graphite calorimetry will improve the robustness of the $\mathrm{D}_{\mathrm{w}}$ primary system [see also ref. 3 , this proceedings collection].

Acknowledgments: The MetrExtRT research program is part of the European Metrology Research program (EMRP), which is jointly funded by the EMRP participating countries within EURAMET and the European Union.

\section{References}

1. ICRU. Report 24: Determination of Absorbed Dose in a Patient Irradiated by Beams of X or Gamma Rays in Radiotherapy Procedures. Bethesda, MD (1976).

2. IAEA. Absorbed dose determination in external beam cancer therapy; An International Code of Practice for Dosimetry Based on Standards of Absorbed Dose to Water. IAEA TRS398, 1-242, Vienna: IAEA (2000).

3. J-M. Bordy, et. al., EMRP Project HLT 09 Metrology for radiotherapy using complex radiation fields, this proceeding;

4. I. S. Sundara Rao, S. B. Naik, Med. Phys. 7 (1983)

5. I. Kawrakow, E. Mainegra-Hing, D.W.O. Rogers, F. Tessier, B.R.B. Walters, The EGSnrc Code System: Monte Carlo simulation of electron and photon transport,
NRC Report PIRS-701 (Ottawa, Canada: National Research Council of Canada (NRC), 2009)

6. F. Hobeila, J. P. Seuntjens, Effect of XCOM photoelectric cross-sections on dosimetric quantities calculated with EGSnrc, Proc. IAEA-CN-96-17: Int. Symp. on Standards and Codes of Practice in Medical Radiation Dosimetry (Vienna) vol 1 (Vienna: IAEA, 2003). 